\title{
State and Output Feedback Nonlinear Model Predictive Control: An Overview
}

\author{
Rolf Findeisen ${ }^{\dagger}$, Lars Imsland ${ }^{\star}$, Frank Allgöwer ${ }^{\dagger *}$, Bjarne A. Foss ${ }^{\star}$ \\ ${ }^{\dagger}$ Institute for Systems Theory in Engineering, University of Stuttgart, \\ 70550 Stuttgart, Germany, \\ \{findeise,allgower\}@ist.uni-stuttgart.de \\ * Department of Engineering Cybernetics, Norwegian University of Science and Technology, \\ 7491 Trondheim, Norway, \\ \{Lars.Imsland,Bjarne.Foss $\} @$ itk.ntnu.no
}

\begin{abstract}
The purpose of this paper is twofold. In the first part we give a review on the current state of nonlinear model predictive control (NMPC). After a brief presentation of the basic principle of predictive control we outline some of the theoretical, computational, and implementational aspects of this control strategy. Most of the theoretical developments in the area of NMPC are based on the assumption that the full state is available for measurement, an assumption that does not hold in the typical practical case. Thus, in the second part of this paper we focus on the output feedback problem in NMPC. After a brief overview on existing output feedback NMPC approaches we derive conditions that guarantee stability of the closed-loop if an NMPC state feedback controller is used together with a full state observer for the recovery of the system state.
\end{abstract}

Keywords: nonlinear model predictive control, output feedback, stability, performance

\section{Introduction}

In many control problems it is desired to design a stabilizing feedback such that a performance criterion is minimized while satisfying constraints on the controls and the states. Ideally one would look for a closed solution for the feedback law satisfying the constraints while optimizing the performance. However, typically the optimal feedback law cannot be found analytically, even in the unconstrained case, since it involves the solution of the corresponding HamiltonJacobi-Bellman partial differential equations. One approach to circumvent this problem is the repeated solution of an open-loop optimal control problem for a given state. The first part of the resulting open-loop input signal is implemented and the whole process is repeated (see Section 2). Control approaches using this strategy are referred to as model predictive control (MPC), moving horizon control or receding horizon control.

In general one distinguishes between linear and nonlinear model predictive control (NMPC). Linear MPC refers to a family of MPC schemes in which linear models are used to predict the system dynamics and considers linear constraints on the states and inputs and a quadratic cost function. Even if the system is linear, the closed-loop dynamics are in general nonlinear due to the presence of constraints. NMPC refers to MPC schemes that are based on nonlinear models and/or consider non-quadratic cost-functionals and general nonlinear constraints on the states and inputs.

Since its first invention in the 70s of the last century, linear MPC has crystallized as one of the key advanced control strategies. By now linear model predictive control, is widely used in industrial applications especially in the process industry, see for example $[38,39,73,77,78]$. The practical success is mainly based on the possibility to take constraints on the states and inputs systematically into account while operating the process optimally.

\footnotetext{
${ }^{*}$ Corresponding author.
} 
Overview on industrial linear MPC techniques can be found in [77] and[78]. In [78] more than 4500 applications spanning a wide range from chemicals to aerospace industries are reported. By now, linear MPC theory can be considered as quite mature. Important issues such as online computation, the interplay between modeling/identification and control and system theoretic issues like stability are well addressed [53,73].

Many systems are, however, inherently nonlinear. Higher product quality specifications and increasing productivity demands, tighter environmental regulations and demanding economical considerations require to operate systems over a wide range of operating conditions and often near the boundary of the admissible region. Under these conditions linear models are often not sufficient to describe the process dynamics adequately and nonlinear models must be used. This inadequacy of linear models is one of the motivations for the increasing interest in nonlinear model predictive control.

The purpose of this paper is twofold. In the first part we provide a review on the current state of NMPC. After a presentation of the basic principle of predictive control we present some of the key theoretical, computational and implementational aspects of this control strategy. We furthermore discuss the inherent advantages and disadvantages of NMPC. Note that this part is not intended to provided a complete review of existing NMPC approaches. For example we mainly focus on NMPC for continuous time systems using sampled measurement information and do not go into details on discrete time NMPC strategies. For more self contained reviews on NMPC we refer to $[3,17,22,69,80]$.

In the second part of the paper the output feedback problem for NMPC is considered. One of the key obstacles of NMPC is that it is inherently a state feedback control scheme using the current state and system model for prediction. Thus, for an application of predictive control in general the full state information is necessary and must be reconstructed from the available state information. However, even if the state feedback NMPC controller and the observer used for the state reconstruction are both stable, there is no guarantee that the overall closed-loop is stable with a reasonable region of attraction, since no general separation principle for nonlinear systems exists. After a review of existing solutions of the output feedback NMPC problem in Section 3.1 we present in Sections 3.2- 3.4 an unifying approach for output feedback NMPC that is based on separation ideas.

In the following, $\|\cdot\|$ denotes the Euclidean vector norm in $\mathbb{R}^{n}$ (where the dimension $n$ follows from context) or the associated induced matrix norm. Vectors are denoted by boldface symbols. Whenever a semicolon ";" occurs in a function argument, the following symbols should be viewed as additional parameters, i.e. $f(x ; \gamma)$ means the value of the function $f$ at $x$ with the parameter $\gamma$.

\section{State Feedback Nonlinear Model Predictive Control}

In this section we provide an up to date overview on the area of state feedback NMPC. Note, however, that we limit the discussion to NMPC for continuous time systems using sampled measurement information. We briefly refer to this as sampled-data NMPC. We do not go into details on NMPC for discrete time systems. However, most of the outlined approaches have dual discrete time counterparts, see for example [3, 22, 69, 80, 81].

\subsection{The Principle of Predictive Control}

Model predictive control is formulated as the repeated solution of a (finite) horizon open-loop optimal control problem subject to system dynamics and input and state constraints. Figure 1 depicts the basic idea behind model predictive control. Based on measurements obtained at time $t$, the controller predicts the dynamic behavior of the system over a prediction horizon $T_{p}$ in the future and determines (over a control horizon $T_{c} \leq T_{p}$ ) the input such that a predetermined open-loop performance objective is minimized. If there were no disturbances and no model-plant mismatch, and if the optimization problem could be solved over an infinite horizon, then the input signal found at $t=0$ could be open-loop applied to the system for all $t \geq 0$. However, due to disturbances, model-plant mismatch, and the finite prediction horizon the actual system behavior is different from the predicted one. To incorporate feedback, the optimal open-loop input is implemented only until the next sampling instant. In principle the time between each new optimization, the sampling time, can vary. We assume for simplicity of presentation, that it is fixed, i.e the optimal control problem is re-evaluated after the constant sampling time $\delta$. Using the new system state at time $t+\delta$, the whole procedure prediction and optimization - is repeated, moving the control and prediction horizon forward. 


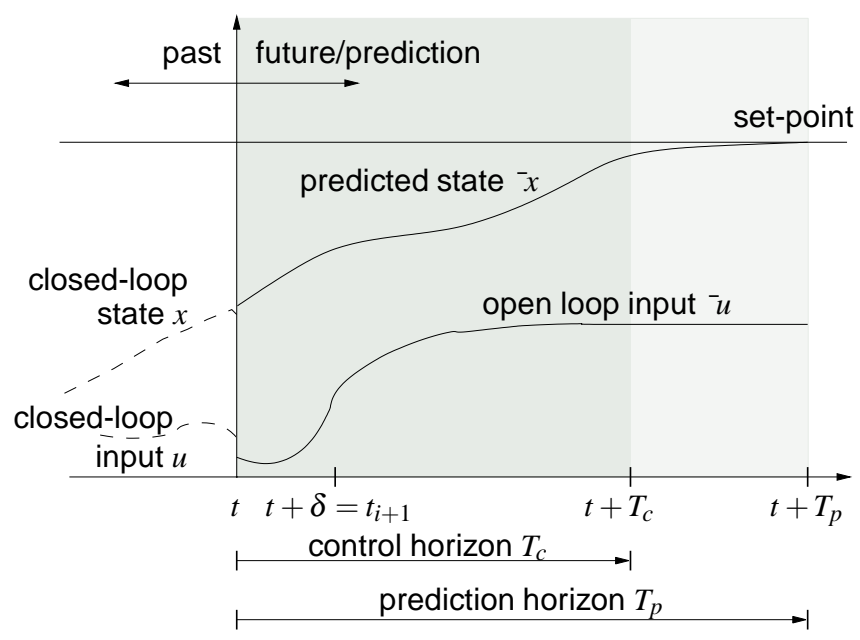

Figure 1: Principle of model predictive control.

In Fig. 1 the open-loop optimal input is depicted as a continuous function of time. To allow a numerical solution of the open-loop optimal control problem the input is often parametrized by a finite number of "basis" functions, leading to a finite dimensional optimization problem. In practice often a piecewise constant input is used, leading to $T_{c} / \delta$ decisions for the input over the control horizon.

Summarizing, a standard NMPC scheme works as follows:

1. Obtain estimates of the states of the system

2. Calculate a constraint-conforming optimal input minimizing the desired cost function over the prediction horizon using the system model and the current state estimate for prediction

3. Implement the first part of the optimal input until the next sampling instant

4. Continue with 1.

While it can be desirable for computational and performance reasons to choose unequal lengths of the prediction and control horizon (see e.g. [60]), we assume in the following that $T_{c}=T_{p}$ for our presentation.

\subsection{Mathematical Formulation of State Feedback NMPC}

Consider the stabilization of time-invariant nonlinear systems of the form

$$
\dot{x}(t)=f(x(t), u(t)), \quad x(0)=x_{0}
$$

subject to the input and state constraints:

$$
\begin{aligned}
& u(t) \in \mathscr{U}, \forall t \geq 0 \\
& x(t) \in \mathscr{X}, \forall t \geq 0 .
\end{aligned}
$$

With respect to the vector field $f: \mathbb{R}^{n} \times \mathbb{R}^{m} \rightarrow \mathbb{R}^{n}$ we assume that it is locally Lipschitz continuous in the region of interest (typically the region of attraction) $)^{1}$ and satisfies $f(0,0)=0$. Furthermore, the set $\mathscr{U} \subset \mathbb{R}^{m}$ is compact, $\mathscr{X} \subseteq \mathbb{R}^{n}$ is connected, and $(0,0) \in \mathscr{X} \times \mathscr{U}$. Typically $\mathscr{U}$ and $\mathscr{X}$ are (convex) box constraints of the form:

$$
\begin{aligned}
\mathscr{U} & :=\left\{u \in \mathbb{R}^{m} \mid u_{\text {min }} \leq u \leq u_{\text {max }}\right\} \\
\mathscr{X} & :=\left\{x \in \mathbb{R}^{n} \mid x_{\text {min }} \leq x \leq x_{\text {max }}\right\},
\end{aligned}
$$

\footnotetext{
${ }^{1}$ This property is needed for the output feedback considerations in Section 3.
} 
with the constant vectors $u_{\min }, u_{\max }$ and $x_{\min }, x_{\max }$.

In sampled-data NMPC an open-loop optimal control problem is solved at discrete sampling instants $t_{i}$ based on the current state information $x\left(t_{i}\right)$. Since we consider a constant sampling time $\delta$, the sampling instants $t_{i}$ are given by $t_{i}=i \cdot \delta, i=0,1,2, \ldots$. When the time $t$ and $t_{i}$ occurs in the same setting, $t_{i}$ should be taken as the closest previous sampling instant $t_{i}<t$.

The open-loop input signal applied in between the sampling instants is given by the solution of the following optimal control problem:

Problem 1: $\quad \min _{\bar{u}(\cdot)} J\left(x\left(t_{i}\right), \bar{u} u(\cdot)\right)$ subject to:

$$
\begin{aligned}
& \left.\dot{\bar{x}} x(\tau)=f\left({ }^{-} x(\tau),{ }^{-} u(\tau)\right), \quad i\right) x\left(x\left(t_{i}\right)\right. \\
& \overline{ }^{u} u(\tau) \in \mathscr{U},{ }^{-} x(\tau) \in \mathscr{X} \quad \tau \in_{i}\left[t_{i}+T_{p}\right] \\
& { }^{-} x\left(t+T_{p}\right) \in \mathscr{E} .
\end{aligned}
$$

The bar denotes predicted variables (internal to the controller), i.e. ${ }^{-} x(\cdot)$ is the solution of (6a) driven by the input $\bar{u}(\cdot):\left[t_{i}, t_{i}+T_{p}\right] \rightarrow \mathscr{U}$ with the initial condition $x\left(t_{i}\right)$. The distinction between the real system variables and the variables in the controller is necessary, since even in the nominal case the predicted values will not be the same as the actual closed-loop values.

The cost functional $J$ minimized over the control horizon $T_{p} \geq \delta>0$ is typically given by

$$
J\left(x\left(t_{i}\right),{ }^{-} u(\cdot)\right):=\int_{t_{i}}^{t_{i}+T_{p}} F\left({ }^{-} x(\tau),{ }^{-} u(\tau)\right) d \tau+E\left({ }^{-} x\left(t T_{p}\right)\right),
$$

where the stage cost $F: \mathscr{X} \times \mathscr{U} \rightarrow \mathbb{R}^{+}$is often assumed to be continuous, satisfies $F(0,0)=0$, and lower bounded by a class $\mathscr{K}$ function ${ }^{2} \alpha_{F}: \alpha_{F}(\|x\|) \leq F(x, u) \forall(x, u) \in \mathscr{X} \times \mathscr{U}$.

The terminal penalty term $E$ and the so called terminal region constraint ${ }^{-} x\left(t+T_{p}\right) \in \mathscr{E}$ might or might not be present. These are often used to enforce nominal stability (see Section 2.3).

The stage cost can for example arise from economical and ecological considerations. Often, a quadratic form for $F$ is used:

$$
F(x, u)=x^{T} Q x+u^{T} R u .
$$

with $Q \geq 0$ and $R>0$.

The state measurement enters the system via the initial condition in (6a) at the sampling instant, i.e. the system model used to predict the future system behavior is initialized by the actual system state. Since all state information is necessary for the prediction, the full state must be either measured or estimated.

The solution of the optimal control problem (6) is denoted by $-\vec{t}\left(\cdot ; x\left(t_{i}\right)\right)$. It defines the open-loop input that is applied to the system until the next sampling instant $t_{i+1}$ :

$$
u\left(t ; x\left(t_{i}\right)\right)=-\vec{t}\left(t ; x\left(t_{i}\right)\right), \quad t \in\left[t_{i}, t_{i+1}\right) .
$$

The control $u\left(t ; x\left(t_{i}\right)\right)$ is a feedback, since it is recalculated at each sampling instant using the new state measurement. In comparison to sampled-data NMPC for continuous time systems, in instantaneous NMPC (also often referred to as receding horizon control) the input is defined by the solution of Problem 1 at all times: $u(x(t))=-t(t ; x(t))$, i.e. no open-loop input is applied, see e.g. [68,69].

The solution of (1) starting at time $t_{1}$ from an initial state $x\left(t_{1}\right)$, applying an input $u:\left[t_{1}, t_{2}\right] \rightarrow \mathbb{R}^{m}$ is denoted by $x\left(\tau ; u(\cdot), x\left(t_{1}\right)\right), \tau \in\left[t_{1}, t_{2}\right]$. We will refer to an admissible input as:

Definition 2.1 (Admissible input) An input $u:\left[0, T_{p}\right] \rightarrow \mathbb{R}^{m}$ for a state $x_{0}$ is called admissible, if it is: a) piecewise continuous, $\left.\left.b) u(\tau) \in \mathscr{U} \forall \tau \in\left[0, T_{p}\right], c\right) x\left(\tau ; u(\cdot), x_{0}\right) \in \mathscr{X} \forall \tau \in\left[0, T_{p}\right], d\right) x\left(T_{p} ; u(\cdot), x_{0}\right) \in \mathscr{E}$.

Furthermore, one refers to the so-called value function as:

Definition 2.2 (Value function) The value function $V(x)$ of the open-loop optimal control Problem 1 is defined as the minimal value of the cost for the state $x: V(x)=J(x,-\vec{u}(\cdot ; x))$.

\footnotetext{
${ }^{2} \mathrm{~A}$ continuous function $\alpha:[0, \infty) \rightarrow[0, \infty)$ is a class $\mathscr{K}$ function, if it is strictly increasing and $\alpha(0)=0$.
} 
The value function plays a central role in the stability analysis of NMPC, since it often serves as a Lyapunov function candidate $[3,69]$.

Typically, no explicit controllability assumption on the system is considered in NMPC. Instead, the stability results are based on the assumption of initial feasibility of the optimal control problem, i.e. the existence of a input trajectory $u(\cdot)$ s.t. all constraints are satisfied.

From a theoretical and practical point of view, one would like to use an infinite prediction and control horizon, i.e. $T_{p}$ and $T_{c}$ in Problem 1 are set to $\infty$. This would lead to a minimization of the cost up to infinity. However, normally the solution of a nonlinear infinite horizon optimal control problem can not be calculated (sufficiently fast). For this reason finite prediction and control horizons are considered. In this case the actual closed-loop input and states will differ from the predicted open-loop ones, even if no model plant mismatch and no disturbances are present. At the sampling instants the future is only predicted over the prediction horizon. At the next sampling instant the prediction horizon moves forward, allowing to obtain more information thus leading to a mismatch of the trajectories.

The unequalness of the predicted and the closed-loop trajectories has two immediate consequences. Firstly, the actual goal to compute a feedback such that the performance objective over the infinite horizon of the closed-loop is minimized is not achieved. In general it is by no means true that the repeated minimization over a moving finite horizon leads to an optimal solution for the infinite horizon problem. The solutions will often differ significantly if a short finite horizon is chosen. Secondly there is in general no guarantee that the closed-loop system will be stable. It is indeed easy to construct examples for which the closed-loop becomes unstable if a short finite horizon is chosen. Hence, when using finite prediction horizons special attention is required to guarantee stability (see Section 2.3).

The summarize the key characteristics and properties of NMPC are:

- NMPC allows the direct use of nonlinear models for prediction.

- NMPC allows the explicit consideration of state and input constraints.

- In NMPC a specified time domain performance criteria is minimized on-line.

- In NMPC the predicted behavior is in general different from the closed loop behavior.

- For the application of NMPC typically a real-time solution of an open-loop optimal control problem is necessary.

- To perform the prediction the system states must be measured or estimated.

Basing the applied input on the solution of an optimal control problem that must be solved on-line is advantageous and disadvantageous at the same time. First, and most important, this allows to directly consider constraints on states and inputs which are often difficult to handle otherwise. Furthermore, the desired cost objective, the constraints and even the system model can in principle be adjusted on-line without making a complete redesign of the controller necessary. However, solving the open-loop optimal control problem, if attacked blindly, can be difficult or even impossible for large systems.

\subsection{State Feedback NMPC and Nominal Stability}

One of the most important questions in NMPC is whether a finite horizon NMPC strategy does guarantee stability of the closed-loop or not. The key problem with a finite prediction and control horizon is due to the difference between the predicted open-loop and the resulting closed-loop behavior. Ideally, one would seek for an NMPC strategy which achieves closed-loop stability independently of the choice of the parameters and, if possible, approximates the infinite horizon NMPC scheme as well as possible. An NMPC strategy that achieves closed-loop stability independently of the choice of the performance parameters is often referred to as an NMPC approach with guaranteed stability. Different approaches to achieve closed-loop stability using finite horizon lengths exist. Here only some central ideas are reviewed and no detailed proofs are given. Moreover, no attempt is made to cover all existing methods. Most of the technical details are left out for reasons of a simple presentation.

Without loss of generality it is assumed that the origin $(x=0$ and $u=0)$ is the steady state to be stabilized.

Infinite Horizon NMPC: Probably the most intuitive way to achieve stability is to use an infinite horizon cost, i.e. $T_{p}$ in Problem 1 is set to $\infty$. In this case the open-loop input and state trajectories computed as the solution of the 
NMPC optimization Problem 1 at a specific sampling instant are in fact equal to the closed-loop trajectories of the nonlinear system due to Bellman's principle of optimality[7]. Thus, the remaining parts of the trajectories at the next sampling instant are still optimal (end pieces of optimal trajectories are optimal). This also implies convergence of the closed-loop. Detailed derivations can be found in [46, 47, 68, 69].

Finite Horizon NMPC Schemes with Guaranteed Stability: Different possibilities to achieve closed-loop stability using a finite horizon length exist. Most of these approaches modify the standard NMPC setup such that stability of the closed-loop can be guaranteed independently of the plant and performance specifications. This is usually achieved by adding suitable equality or inequality constraints and suitable additional penalty terms to the standard setup. The additional terms are generally not motivated by physical restrictions or performance requirements but have the sole purpose to enforce stability. Therefore, they are usually called stability constraints.

One possibility to enforce stability with a finite prediction horizon is to add the so called zero terminal equality constraint at the end of the prediction horizon, i.e.

$$
-x(t+T)=0
$$

is added to Problem $1[15,47,68,70]$. This leads to stability of the closed-loop, if the optimal control problem has a solution at $t=0$. Similar to the infinite horizon case the feasibility at one sampling instant does imply feasibility at the following sampling instants and a decrease in the value function. One disadvantage of a zero terminal constraint is that the predicted system state is forced to reach the origin in finite time. This leads to feasibility problems for short prediction/control horizon lengths, i.e. to small regions of attraction. From a computational point of view, an exact satisfaction of a zero terminal equality constraint does require in general an infinite number of iterations in the optimization and is thus not desirable. The main advantages of a zero terminal constraint are the straightforward application and the conceptual simplicity.

Many schemes exist that try to overcome the use of a zero terminal constraint of the form (10). Most of them use the terminal region constraint ${ }^{-} x(t+T) \in \mathscr{E}$ and/or a terminal penalty term $E\left({ }^{-} x(t+T)\right)$ to enforce stability and feasibility. Typically the terminal penalty $E$ and the terminal region $\mathscr{E}$ are determined off-line such that the cost function

$$
J\left(x(t),{ }^{-} u(\cdot)\right)=\int_{t_{i}}^{t_{i}+T_{p}} F\left({ }^{-} x(\tau),{ }^{-} u(\tau)\right) d \tau+E\left({ }^{-} x\left(t \frac{1}{p}\right) \overline{)}\right.
$$

gives an upper bound on the infinite horizon cost and guarantees a decrease in the value function as the horizon recedes in time.

We do not go into details about the different approaches. Instead we state the following theorem, which gives conditions for the convergence of the closed-loop states to the origin. It is a slight modification of the results given in [36] and [16,17].

\section{Theorem 2.1 (Stability of sampled-data NMPC) Suppose that}

a) the terminal region $\mathscr{E} \subseteq \mathscr{X}$ is closed with $0 \in \mathscr{E}$ and that the terminal penalty $E(x) \in C^{1}$ is positive semi-definite

(b) the terminal region and terminal penalty term are chosen such that $\forall x \in \mathscr{E}$ there exists an (admissible) input $u_{\mathscr{E}}:[0, \delta] \rightarrow \mathscr{U}$ such that $x(\tau) \in \mathscr{E} \forall \tau \in[0, \delta]$ and

$$
\frac{\partial E}{\partial x} f\left(x(\tau), u_{\mathscr{E}}(\tau)\right)+F\left(x(\tau), u_{\mathscr{E}}(\tau)\right) \leq 0 \quad \forall \tau \in[0, \delta]
$$

(c) the NMPC open-loop optimal control problem is feasible for $t=0$.

Then in the closed-loop system (1) with (9) $x(t)$ converges to the origin for $t \rightarrow \infty$, and the region of attraction $\mathscr{R}$ consists of the states for which an admissible input exists.

Proof. The proof is given here for sake of completeness. It bases on using the value function as a decreasing Lyapunovlike function. As usual in predictive control the proof consists of two parts: in the first part it is established that initial feasibility implies feasibility afterwards. Based on this result it is then shown that the state converges to the origin. 
Feasibility: Consider any sampling instant $t_{i}$ for which a solution exists (e.g. $\left.t_{0}\right)$. In between $t_{i}$ and $t_{i+1}$ the optimal input $-\vec{a}\left(\cdot ; x\left(t_{i}\right)\right)$ is implemented. Since no model plant mismatch nor disturbances are present, $x\left(t_{i+1}\right)=$ ${ }^{-} x\left(t_{t_{1}} ;{ }^{-\imath}\left(\cdot ; x\left(t_{i}\right)\right), x\left(t_{i}\right)\right)$. The remaining piece of the optimal input $-\grave{a}\left(\tau ; x\left(t_{i}\right)\right), \tau \in\left[t_{i+1}, t_{i}+T_{p}\right]$ satisfies the state and input constraints. Furthermore, ${ }^{-} x\left(t+T_{p} ; x\left(t_{i}\right),{ }^{-} \vec{t}\left(\cdot ; x\left(t_{i}\right)\right)\right) \in \mathscr{E}$ and we know from Assumption (b) of the theorem that for all $x \in \mathscr{E}$ there exists at least one input $u_{\mathscr{E}}(\cdot)$ that renders $\mathscr{E}$ invariant over $\delta$. Picking any such input we obtain as admissible input for any time $t_{i}+\sigma, \sigma \in\left(0, t_{i+1}-t_{i}\right]$

$$
\tilde{u}\left(\tau ; x\left(t_{i}+\sigma\right)\right)=\left\{\begin{array}{l}
-\tilde{u}\left(\tau ; x\left(t_{i}\right)\right), \tau \in\left[t_{i}+\sigma, t_{i}+T_{p}\right] \\
u_{\mathscr{E}}\left(\tau-t_{i}-T_{p}\right), \tau \in\left(t_{i}+T_{p}, t_{i}+T_{p}+\sigma\right]
\end{array} .\right.
$$

Specifically, we have for the next sampling time $\left(\sigma=t_{i+1}-t_{i}\right)$ that $\tilde{u}\left(\cdot ; x\left(t_{i+1}\right)\right)$ is a feasible input, hence feasibility at time $t_{i}$ implies feasibility at $t_{i+1}$. Thus, if (6) is feasible for $t=0$, it is feasible for all $t \geq 0$.

Furthermore, if the states for which an admissible input exists converge to the origin, it is clear that the region $\mathscr{R}$ that consists of those points belongs to the region or attraction.

Convergence: We first show that the value function is decreasing starting from a sampling instant. Remember that the value of $V$ at $x\left(t_{i}\right)$ is given by:

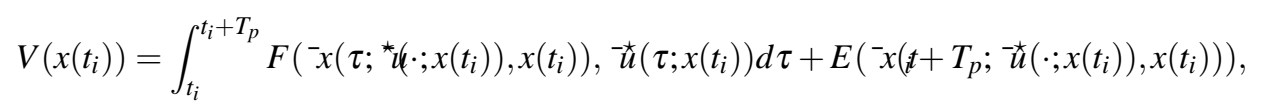

and the cost resulting from (13) starting from any $x\left(t_{i}+\sigma ;{ }^{-\imath}\left(\cdot ; x\left(t_{i}\right)\right), x\left(t_{i}\right)\right), \sigma \in\left(0, t_{i+1}-t_{i}\right]$, using the input $\tilde{u}\left(\tau, x\left(t_{i}+\right.\right.$ $\sigma)$ ), is given by:

$$
\begin{aligned}
J\left(x\left(t_{i}+\sigma\right), \tilde{u}\left(\cdot ; x\left(t_{i}+\sigma\right)\right)\right)=\int_{t_{i}+\sigma}^{t_{i}+\sigma+T_{p}} F\left({ }^{-} x\left(\tau ; \tilde{u}(\cdot ; x(t+\sigma)), x\left(t_{i}+\sigma\right)\right), \tilde{u}\left(\tau ; x\left(t_{i}+\sigma\right)\right)\right) d \tau \\
+E\left({ }^{-} x\left(i t+\sigma+T_{p} ; \tilde{u}\left(\cdot ; x\left(t_{i}+\sigma\right)\right), x\left(t_{i}+\sigma\right)\right)\right) .
\end{aligned}
$$

Reformulation yields

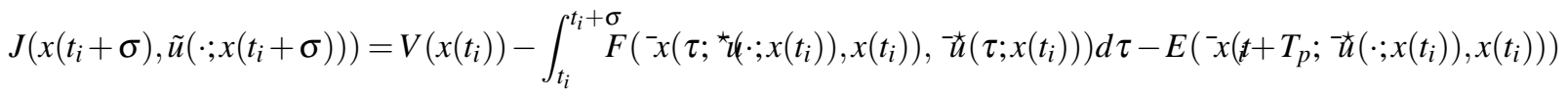

$$
\begin{aligned}
& +\int_{t_{i}+T_{p}}^{t_{i}+\sigma+T_{p}} F\left({ }^{-} x\left(\tau ; \tilde{u}(\cdot ; x(\boldsymbol{t}+\sigma)), x\left(t_{i}+\sigma\right)\right), \tilde{u}\left(\tau ; x\left(t_{i}+\sigma\right)\right)\right) d \tau+E\left({ }^{-} x\left(\boldsymbol{t}+\sigma+T_{p} ; \tilde{u}\left(\cdot, x\left(t_{i}+\sigma\right)\right), x\left(t_{i}+\sigma\right)\right)\right) .
\end{aligned}
$$

Integrating inequality (12) from $t_{i}+\sigma$ to $t_{i}+\sigma+T_{p}$ starting from $x\left(t_{i}+\sigma\right)$ we obtain zero as an upper bound for the last three terms on the right side. Thus,

$$
J\left(x\left(t_{i}+\sigma\right), \tilde{u}\left(\cdot, x\left(t_{i}+\sigma\right)\right)\right)-V\left(x\left(t_{i}\right)\right) \leq-\int_{t_{i}}^{t_{i}+\sigma} F\left({ }^{-} x\left(\tau ;{ }^{\star} u\left(\cdot ; x\left(t_{i}\right)\right)\right),{ }^{-} \vec{u}\left(\tau ; x\left(t_{i}\right)\right)\right) d \tau .
$$

Since $\tilde{u}$ is only a feasible but not necessarily the optimal input for $x\left(t_{i}+\sigma\right)$, it follows that

$$
V\left(x\left(t_{i}+\sigma\right)\right)-V\left(x\left(t_{i}\right)\right) \leq-\int_{t_{i}}^{t_{i}+\sigma} F\left(-x\left(\tau ;{ }^{*}\left(x \cdot ; x\left(t_{i}\right)\right), x\left(t_{i}\right)\right),-{ }^{-\star}\left(\tau ; x\left(t_{i}\right)\right)\right) d \tau,
$$

i.e. the value function is decreasing along solution trajectories starting at a sampling instant $t_{i}$. Especially we have that:

$$
V\left(x\left(t_{i+1}\right)\right)-V\left(x\left(t_{i}\right)\right) \leq-\int_{t_{i}}^{t_{i+1}} F\left({ }^{-} x\left(\tau ;{ }^{\star}\left(c \cdot ; x\left(t_{i}\right)\right), x\left(t_{i}\right)\right),-{ }^{\star} t\left(\tau ; x\left(t_{i}\right)\right)\right) d \tau .
$$

By assumption, this decrease in the value function is strictly positive for $x\left(t_{i}\right) \neq 0$. Since this holds for all sampling instants, convergence can be established as in $[18,36]$ by an induction argument and the application of Barbalat's lemma. 
Loosely speaking, $E$ is an $F$-conform local control Lyapunov function in $\mathscr{E}$. The terminal region constraint enforces feasibility at the next sampling instant and allows, similarly to the infinite horizon case, to show that the value function is strictly decreasing. Thus stability can be established. Note that this result is nonlocal in nature, i.e. there exists a region of attraction $\mathscr{R}$ which is of at least the size of $\mathscr{E}$. Typically the region of attraction resulting from this scheme is much larger than $\mathscr{E}$ and contains the set of all states for which the open-loop optimal control problem has a feasible solution.

Various ways to determine a suitable terminal penalty term and terminal region exist. Examples are the use of a control Lyapunov function as terminal penalty $E[45,75,84]$ or the use of a local nonlinear or linear control law to determine a suitable terminal penalty $E$ and a terminal region $\mathscr{E}[17,18,20,63,71]$.

Quasi-infinite horizon NMPC: We exemplify the choice of a suitable terminal region constraint and a terminal penalty term considering the so called quasi-infinite horizon NMPC (QIH-NMPC) approach [16,18,31]. In QIHNMPC $E$ and $\mathscr{E}$ are obtained on the basis of a locally stabilizing linear control law $u_{\mathscr{E}}=K x$ considering a quadratic stage cost of the form (8). The terminal penalty term $E$ in this case is quadratic and of the form $E(x)=x^{T} P x$. The following procedure gives a systematic approach to compute the terminal region and a terminal penalty matrix off-line [18], assuming that the Jacobian linearization $(A, B)$ of (1) is stabilizable, where $A:=\frac{\partial f}{\partial x}(0,0)$ and $B:=$ $\frac{\partial f}{\partial u}(0,0)$.

Step 1: Solve the linear control problem based on the Jacobian linearization $(A, B)$ of (1) to obtain a locally stabilizing linear state feedback $u_{\mathscr{E}}=K x$.

Step 2: Define $A_{K}:=A+B K$, and choose a constant $\kappa \in[0, \infty)$ satisfying $\kappa<-\lambda_{\max }\left(A_{K}\right)$ and solve the Lyapunov equation

$$
\left(A_{K}+\kappa I\right)^{T} P+P\left(A_{K}+\kappa I\right)=-\left(Q+K^{T} R K\right)
$$

to get a positive definite and symmetric $P$.

Step 3 : Find the largest possible $\alpha_{1}$ defining a region

$$
\mathscr{E}_{1}:=\left\{x \in \mathbb{R}^{n} \mid x^{T} P x \leq \alpha_{1}\right\}
$$

such that $K x \in \mathscr{U}$, for all $x \in \mathscr{E}_{1}$.

Step 4 : Find the largest possible $\alpha \in\left(0, \alpha_{1}\right]$ specifying a terminal region

$$
\mathscr{E}:=\left\{x \in \mathbb{R}^{n} \mid x^{T} P x \leq \alpha\right\}
$$

such that the optimal value of the following optimization problem is non-positive:

$$
\max _{x}\left\{x^{T} P \varphi(x)-\kappa \cdot x^{T} P x \mid x^{T} P x \leq \alpha\right\}
$$

where $\varphi(x):=\mathbf{f}(x, K x)-A_{K} x$.

This procedure allows to calculate $E$ and $\mathscr{E}$ if the linearization of the system at the origin is stabilizable. If the terminal penalty term and the terminal region are determined accordingly, the open-loop optimal trajectories found at each time instant approximate the optimal solution for the infinite horizon problem.

Remark 2.1 Theorem 2.1 allows to consider the stabilization of systems that can only be stabilized by feedback that is discontinuous in the state [36,37], e.g. nonholonomic mechanical systems. This is possible since the optimal input signal is applied over the sampling time open-loop to the system. For such systems, however, it is in general rather difficult to determine a suitable terminal region and a terminal penalty term - for instance, the QIH-NMPC method cannot be used. 
Remark 2.2 The use of a terminal inequality constraint leads to computational and feasibility advantages compared to the infinite horizon and zero terminal constraint approach. No zero terminal constraint must be met in finite time. The solution time necessary for solving the open-loop optimal control problem is decreased, since no "boundaryvalue" problem stemming from the zero terminal constraint must be solved. Furthermore, note that in NMPC it is in general not necessary to find always an optimal solutions of Problem 1 in order to guarantee stability [18, 45, 82]. Only a feasible solution leading to a decrease in the value function is necessary. This can be utilized to decrease the necessary on-line solution time and makes the practical application more robust.

Summarizing, the nominal stability question of NMPC in the state feedback case is well understood. Various NMPC approaches that guarantee stability exist.

\subsection{Robustness and NMPC}

The NMPC schemes presented are based on the assumption that the actual system is identical to the model used for prediction, i.e. that no model/plant mismatch or unknown disturbances are present. Clearly, this is very unrealistic for practical applications and the development of an NMPC framework to address robustness issues is of paramount importance. In general one distinguishes between the inherent robustness properties of NMPC and NMPC designs taking the uncertainty/disturbances directly into account.

The inherent robustness of NMPC is related to the fact that nominal NMPC can cope with uncertainties and disturbances without taking them directly into account. This fact stems from the close relation of NMPC to optimal control and inverse optimality considerations $[15,64]$. Without going into details, we refer to $[64,69,71,83]$.

NMPC schemes that take the uncertainty/disturbance directly into account are often based on min-max considerations. A series of different approaches can be distinguished. For details we refer for example to $[13,19,49,52,58,61$, 62].

\subsection{Effi cient Real-time Solution of NMPC}

One important precondition for the application of NMPC, is the availability of reliable and efficient numerical dynamic optimization algorithms, since at every sampling time a nonlinear dynamic optimization problem must be solved in real-time. Solving such an optimization problem efficiently and fast is, however, not a trivial task and has attracted strong research interest in recent years (see e.g. [6, 10, 11, 23, 25-27, 55, 65-67, 86, 90]).

Typically so called direct solution methods $[11,12,76]$ are used, i.e. the original infinite dimensional problem is turned into a finite dimensional one by discretizing the input (and also possibly the state). Basically this is done by parameterizing the input (and possibly the states) by a finite number of parameters and to solve/approximate the differential equations during the optimization. We do not go into further details here and instead refer to [12, 25, 67].

Recent studies have shown, that using special dynamic optimizers and tailored NMPC schemes allows to employ NMPC to practically relevant problems (see e.g. [6, 27, 32, 66, 86],[35]) even with todays computational power.

\section{NMPC Output Feedback Problem}

So far we assumed that the full state information can be measured and is available as initial condition for predicting the future system behavior. In many applications, however, the system state can not be fully measured, i.e. only an output $y$ is directly available for feedback:

$$
y=h(x, u)
$$

where $y(t) \in \mathbb{R}^{p}$ are the measured outputs and where $h: \mathbb{R}^{n} \times \mathbb{R}^{m} \rightarrow \mathbb{R}^{p}$ maps the state and input to the output. Thus, to apply predictive control methods the state must be estimated from the measured outputs using suitable state observers or filters. However, even if the state feedback NMPC controller and the observer used are both stable, there is no guarantee that the overall closed loop is stable with a reasonable region of attraction, since no general valid separation principle for nonlinear systems exists. 
To achieve non-local stability results of the observer based output feedback NMPC controller, two possibilities (and mixtures thereof) seem to be attractive:

Separated Designs/Certainty Equivalence Approach: In this approach the observer state estimate is used as the real system state following the "certainty equivalence" principle. To show stability of the closed-loop, one tries to separate the observer error from the state feedback for example by time scale separation. Utilizing observers for which the speed of convergence of the observer error can be made sufficiently fast and the absolute achieved observer error can be made sufficiently small, semi-regional stability results for the closed-loop can be established.

Consideration of the observer error in the NMPC controller: In this approach the observer error is acknowledged in the controller, typically by using some bounds on the observer error. This solution is closely related to the design of robustly stabilizing NMPC schemes and typically requires observers that deliver an estimate of the observer error.

Following the first approach we derive in this section, for a broad class of state feedback nonlinear model predictive controllers, conditions on the observer that guarantee that the closed-loop is semi-globally practically stable. The result is based on the results presented in [34,43], where high-gain observers are used for state recovery. Basically we exploit that sampled-data predictive controllers that possess a continuous value function are inherently robust to small disturbances, i.e. we will consider the estimation error as a disturbance acting on the closed-loop. Before we derive the approach, we give a brief review of the existing output feedback NMPC approaches.

\subsection{Existing Output-Feedback Results}

Various researchers have addressed the question of output feedback NMPC using observers for state recovery. We restrict the discussion to output feedback model predictive control schemes relying on state space models for prediction and differentiate between the two output feedback design approaches as outlined above. The "certainty equivalence"method is often used in a somewhat ad-hoc manner in industry [78], e.g. based on the (extended) Kalman filter as a state observer. In the presence of a separation principle, this would be a theoretically sound way to achieve a stabilizing output feedback scheme. Unfortunately, a general separation principle does not exist for MPC - even in the case of linear models, the separation principle for linear systems is void due to the presence of constraints. Thus, at the outset, nothing can be said about closed loop stability in this case, and it seems natural that one has to restrict the class of systems one considers to obtain results. As an example, [91] shows global asymptotic stability for the special case of discrete-time linear open-loop stable systems.

For a more general class of nonlinear systems, it can be shown that the properties of the value function as a Lyapunov function gives some robustness of NMPC to "small" estimation errors. For "weakly detectable" discretetime systems, this was first pointed out in [83] (see also [57, 59], and an early version in [74]). However, these results must be interpreted as "local", in the sense that even though that an approximated region of attraction can be calculated in principle, it is not clear how parameters in the controller or observer must be tuned to influence the size of the region of attraction.

In [24], local uniform asymptotic stability of contractive NMPC in combination with a "sampled" EKF state estimator is established.

Non-local results are obtained in [72], where an optimization based moving horizon observer combined with the NMPC scheme proposed in [71] is shown to lead to (semi-global) closed-loop stability. For the results to hold, however, a global optimization problem for the moving horizon observer with an imposed contraction constraint must be solved.

More recently, "regional" separation principle-based approaches have appeared for a wide class of NMPC schemes. In $[43,44]$ it was shown that based on the results of $[5,85]$, semi-global practical stability results could be obtained for instantaneous NMPC based on a special class of continuous-time models, using high gain observers for state estimation. In this context, semi-global practical stability means that for any compact region inside the state feedback NMPC region of attraction, there exists a sampling time and an observer gain such that for system states starting in this region, the closed loop take the state into any small region containing the origin. The result of [43] are developed further to the more realistic sampled-data case in [33,34], still considering a class (albeit a larger one) of continuoustime systems. In [30] it is pointed out how these results can be seen as a consequence of NMPC state feedback robustness. In [42], conditions are given on the system and the observer for the state to actually converge to the origin. 
Related results appeared recently in [1], where for the same system class as considered in [43], semi-global practical stability results are given for sampled-data systems using sampled high-gain observers.

In [89] a scheduled state feedback NMPC scheme is combined with an exponential convergent observer, and regional stability results are established. On a related note, the same authors show in [88] how an NMPC controller can be combined with a convergent observer to obtain stability, where stability is taken care of off-line.

In the robust design approach the errors in the state estimate are directly accounted for in the state feedback predictive controller. For linear systems, [8] introduces a set membership estimator to obtain quantifiable bounds on the estimation error, which are used in a robust constraint-handling predictive controller. The setup of [8] is taken further in [21], using a more general observer, and considering more effective computational methods. For the same class of systems, [56] does joint estimation and control calculation based on a minimax formulation, however without obtaining stability guarantees.

For linear systems with input constraints, the method in [54] obtains stability guarantees through computation of invariant sets for the state vector augmented with the estimation error. In a similar fashion, by constructing invariant sets for the observer error, [50] adapts the NMPC controller in [14] such that the total closed loop is asymptotically stable.

\subsection{Output Feedback NMPC with Stability-Setup}

In the following we present one specific approach to output feedback NMPC. It is based on the fact that sampled-data predictive controllers that possess a continuous value function are inherently robust to small disturbances, i.e. we will consider the estimation error as a disturbance acting on the closed-loop. This inherent robustness property of NMPC is closely connected to recent results on the robustness properties of discontinuous feedback via sample and hold [48]. However, here we consider the specific case of sampled-data NMPC controller and we do not assume that the applied input is realized via a hold element.

Setup: Instead of the real system state $x\left(t_{i}\right)$ at every sampling instant only a state estimate $\hat{x}\left(t_{i}\right)$ is available. Thus, instead of the optimal feedback (9) the following "disturbed" feedback is applied:

$$
u\left(t ; \hat{x}\left(t_{i}\right)\right)=-\vec{u}\left(t ; \hat{x}\left(t_{i}\right)\right), \quad t \in\left[t_{i}, t_{i+1}\right) .
$$

Note that the estimated state $\hat{x}\left(t_{i}\right)$ can be outside the region of attraction $\mathscr{R}$ of the state feedback NMPC controller. To avoid feasibility problems we assume that in this case the input is fixed to an arbitrary, but bounded value.

The NMPC scheme used for feedback is assumed to fit the setup of Theorem 2.1. Additionally, we make the following assumptions:

Assumption 1 In the nominal region of attraction $\mathscr{R} \subseteq \mathscr{X} \subseteq \mathbb{R}^{n}$ the following holds:

1. Along solution trajectories starting at a sampling instant $t_{i}$ at $x\left(t_{i}\right) \in \mathscr{R}$, the value function satisfies for all positive $\tau$ :

$$
V\left(x\left(t_{i}+\tau\right)\right)-V\left(x\left(t_{i}\right)\right) \leq-\int_{t_{i}}^{t_{i}+\tau} F\left(x(s), u\left(s ; x\left(s_{i}\right)\right)\right) d s .
$$

2. The value function $V(x)$ is uniformly continuous.

3. For all compact subsets $\mathscr{S} \subset \mathscr{R}$ there is at least one level set $\Omega_{c}=\{x \in \mathscr{R} \mid V(x) \leq c\}$ s.t. $\mathscr{S} \subset \Omega_{c}$.

Following Theorem 2.1, Assumption 1.1 imply stability of the state feedback NMPC scheme (compare equation (18) in the proof of Theorem 2.1), and is typically satisfied for stabilizing NMPC schemes. However, in general there is no guarantee that a stabilizing NMPC schemes satisfies Assumption 1.2 and 1.3, especially if state constraints are present. As is well known [36,40,70], NMPC can also stabilize systems that cannot be stabilized by feedback that is continuous in the state. This in general also implies a discontinuous value function. Many NMPC schemes, however, satisfy this assumption at least locally around the origin [18, 20,69]. Furthermore, for example NMPC schemes that are based on control Lyapunov functions [45] and that are not subject to constraints on the states and inputs satisfy Assumption 1. 
Remark 3.1 Note that the uniform continuity assumption on $V(x)$ implies that for any compact subset $\mathscr{S} \subset \mathscr{R}$ there exists a $\mathscr{K}$-function $\alpha_{V}$ such that for any $x_{1}, x_{2} \in \mathscr{S}\left\|V\left(x_{1}\right)-V\left(x_{2}\right)\right\| \leq \alpha_{V}\left(\left\|x_{1}-x_{2}\right\|\right)$.

We do not state any explicit observability assumptions, since they depend on the observer used for state recovery. As outlined later, several different observers satisfy the conditions required for the semi-global practical stability results to be derived.

Concerning the observer used, however, we assume that after an initial phase, the observer error at the sampling instants can be made sufficiently small, i.e. we assume that

Assumption 2 (Observer error convergence) For any desired maximum estimation error $e_{\max }>0$ there exist observer parameters, such that

$$
\left\|x\left(t_{i}\right)-\hat{x}\left(t_{i}\right)\right\| \leq e_{\max }, \quad \forall t_{i} \geq k_{\mathrm{conv}} \delta .
$$

Here $k_{\text {conv }}>0$ is a freely chosen, but fixed number of sampling instants after which the observer error has to satisfy (27).

Remark 3.2 Depending on the observer used, further conditions on the system (e.g. observability assumptions) might be necessary. Note that the observer does not have to operate continuously since the state information is only necessary at the sampling times.

Note that there exist a series of observers which satisfy Assumption 2, see Section 3.4. Examples are high-gain observers and moving horizon observers with contraction constraint.

Since we do not assume that the observer error converges to zero, we can certainly not achieve asymptotic stability of the origin, nor can we render the complete region of attraction of the state feedback controller invariant. Thus, we consider in the following the question if the system state in the closed loop can be rendered semi-globally practically stable, under the assumption that for any maximum error $e_{\max }$ there exist observer parameters such that (27) holds. In this context semi-globally practically stable means, that for arbitrary sets $\Omega_{\alpha} \subset \Omega_{c_{0}} \subset \Omega_{c} \subset \mathscr{R}, 0<\alpha<c_{0}<c$ there exist observer parameters and a maximum sampling time $\delta$ such that $\forall x(0) \in \Omega_{c_{0}}: 1 . x(t) \in \Omega_{c}, t>0,2 . \exists T_{\alpha}>0$ s.t. $x(t) \in \Omega_{\alpha}, \forall t \geq T_{\alpha}$. For clarification see Fig. 2.

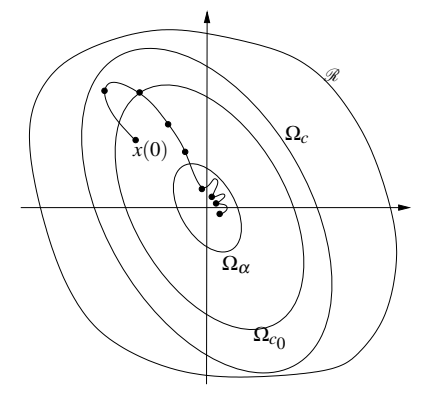

Figure 2: Set of initial conditions $\Omega_{c_{0}}$, desired maximum attainable set $\Omega_{c}$ and desired region of convergence $\Omega_{\alpha}$

Note, that in the following we only consider level sets for the desired set of initial conditions $\left(\Omega_{c_{0}}\right)$, the maximum attainable set $\left(\Omega_{c}\right)$ and the set of desired convergence $\left(\Omega_{\alpha}\right)$. We do this for pure simplification of the presentation. In principle one can consider arbitrary compact sets which contain the origin, and subsets of each other and of $\mathscr{R}$, since due to Assumption 1.3 it is always possible to find suitable covering level sets.

Basic Idea: The derived results are based on the observation that small state estimation errors lead to a (small) difference between the predicted state trajectory ${ }^{-} x$ based on the estimated state $\hat{x}(t)$ and the real state trajectory (as long as both of them are contained in the set $\Omega_{c}$ ). As will be shown, the influence of the estimation error (after the convergence time $k_{\mathrm{conv}} \delta$ ) of the observer can in principle be bounded by

$$
V\left(x\left(t_{i}\right)\right)-V\left(x\left(t_{i+1}\right)\right) \leq \mathrm{e}_{\mathrm{r}}\left(\left\|x\left(t_{i}\right)-\hat{x}\left(t_{i}\right)\right\|\right)-\int_{t_{i}}^{t_{i+1}} F\left(x\left(\tau ; u^{*}\left(\cdot ; \hat{x}\left(t_{i}\right)\right), x\left(t_{i}\right)\right), u^{*}\left(\tau ; \hat{x}\left(t_{i}\right)\right)\right) d \tau,
$$


where $e_{r}$ corresponds to the state estimation error contribution. Note that the integral contribution is strictly negative. Thus, if $e_{r}$ "scales" with the size of the observer error (it certainly also scales with the sampling time $\delta$ ) one can achieve contraction of the value function.

However, considering Assumption 2, the bounding is possible after a certain time. To avoid that the system state leaves the set $\Omega_{c}$ during this time we have to decrease the sampling time $\delta$ (or use smaller sampling times initially).

In order to bound the integral contribution on the right side of (28), we state the following fact:

Fact 1 For any $c>\alpha>0$ with $\Omega_{c} \subset \mathscr{R}, T_{p}>\delta>0$ the lower bound $V_{\min }(c, \alpha, \delta)$ on the value function exists and is non-trivial for all $x_{0} \in \Omega_{c} / \Omega_{\alpha}: 0<V_{\min }(c, \alpha, \delta):=\min _{x_{0} \in \Omega_{c} / \Omega_{\alpha}} \int_{0}^{\delta} F\left({ }^{-} x\left(s ;{ }^{*}\left(c \cdot ; x_{0}\right), x_{0}\right), \quad \breve{u}\left(s ; x_{0}\right)\right) d s<\infty$.

\subsection{Output Feedback NMPC with Stability-Main Result}

Under the given setup the following theorem holds

Theorem 3.1 Assume that Assumption 1 and 2 hold. Then given arbitrary level sets $\Omega_{\alpha} \subset \Omega_{c_{0}} \subset \Omega_{c} \subset \mathscr{R}$, there exists a maximum allowable observer error $e_{\max }$ and a maximum sampling time $\delta_{\max }$, such that for all initial conditions $x_{0} \in \Omega_{c_{0}}$ the state $x(\tau)$ stays in the region $\Omega_{c}$ and converges in finite time to the set $\Omega_{\alpha}$.

Proof. The proof is divided in three parts. In the first part it is ensured that the system state does not leave the maximum admissible set $\Omega_{c}$ during the convergence time $k_{\mathrm{conv}} \delta$ of the observer. This is achieved by decreasing the sampling time $\delta$ sufficiently. In the second part is shown, that by requiring a sufficiently small $e_{\text {max }}$ the system state converges into the set $\Omega_{\alpha / 2}$. In the third part it is shown that the state will not leave the set $\Omega_{\alpha}$ once it has entered $\Omega_{\alpha / 2}$ at a sampling time.

First part $\left(x(\tau) \in \Omega_{c} \forall x(0) \in \Omega_{c_{0}}, \tau \in\left[0, k_{\text {conv }} \delta\right)\right)$ :

We first derive conditions guaranteeing that states starting in $\Omega_{0}$ stay in $\Omega_{c}$ for a certain time for all possible inputs. Note that $\Omega_{c_{0}}$ is strictly contained in $\Omega_{c}$ and thus also in $\Omega_{c_{1}}$, with $c_{1}:=c_{0}+\left(c-c_{0}\right) / 2$. Thus, there exists a time $T_{c_{0} c_{1}}$ such that $x(\tau) \in \Omega_{c_{1}}, \forall 0 \leq \tau \leq T_{c_{0} c_{1}}$. The existence of such a time is guaranteed, since as long as $x(\tau) \in \Omega_{c}$, $\|x(\tau)-x(0)\| \leq \int_{0}^{\tau}\|f(x(s), u(s))\| d s \leq k_{\Omega_{c}}$ t where $k_{\Omega_{c}}$ is a constant depending on the Lipschitz constants of $f$ and on the bounds on $u$. We take $T_{c_{0} c_{1}}$ as the smallest (worst case) time to reach the boundary of $\Omega_{c_{1}}$ from any point $x_{0} \in \Omega_{c_{0}}$ allowing $u(s)$ to take any value in $\mathscr{U}$. By similar arguments there exists also a time $T_{c_{2} c}$ such that for all $x\left(t_{i}\right) \in \Omega_{c_{2}}$, $x(\tau) \in \Omega_{c}, \forall \tau \in\left[t_{i}, t_{i}+T_{c_{2} c}\right)$ where $c_{2}=c_{1}+\left(c-c_{1}\right) / 2$. We pick now the maximum sampling time $\delta_{\max }$ as

$$
\delta_{\max } \leq \min \left\{T_{c_{0} c_{1}} / k_{\text {conv }}, T_{c_{2} c}\right\} .
$$

The sampling time $\delta$ is assumed to be less or equal to this maximum value for the remainder of the proof. This ensures that the state will be at least during the convergence of the observer completely contained in $\Omega_{c}$.

Furthermore we need for the second part of the proof that for any $x\left(t_{i}\right) \in \Omega_{c_{1}} \hat{x}\left(t_{i}\right) \in \Omega_{c_{2}}$ after the observer has converged. Note that due to Assumption 2 there always exist observer parameters such that after $\delta_{\max } k_{\text {conv }}$ the observer error is smaller than any desirable $e_{\max }$. If we require that

$$
\alpha_{V}\left(e_{\max }\right) \leq c_{2}-c_{1}
$$

it is guaranteed that $\hat{x}\left(t_{i}\right) \in \Omega_{c_{2}}$ if $x\left(t_{i}\right) \in \Omega_{c_{1}}$.

Second part (decrease of the value function after observer convergence and finite time convergence to $\Omega_{\alpha / 2}$ ):

We assume that $x\left(t_{i}\right) \in \Omega_{c_{1}}$. For simplicity of notation, $u_{\gamma_{x}}$ denotes the optimal input resulting from $\hat{x}\left(t_{i}\right)$ and $u_{x}$ denotes the input that correspond to the real state $x\left(t_{i}\right)$. Furthermore, $x_{i}=x\left(t_{i}\right)$ and $\hat{x}_{i}=\hat{x}\left(t_{i}\right)$.

Note that since $x_{i} \in \Omega_{c_{1}}$ we know by the derivations in the first part of the proof that $\hat{x}_{i} \in \Omega_{c_{2}}$ and that $x(\tau) \in \Omega_{c}$, $x\left(\tau ; \hat{x}_{i}, u_{\lambda}\right) \in \Omega, x\left(\tau ; x_{i}, u_{\lambda}\right) \in \Omega_{c} \forall \tau \in\left[t_{i}, t_{i+1}\right)$. Under these conditions the following equality is valid:

$$
V\left(x\left(\tau ; x_{i}, u_{\hat{y}}\right)\right)-V\left(x_{i}\right)=V\left(x\left(\tau ; x_{i}, u_{\lambda}\right)\right)-V\left(x\left(\tau ; \hat{x}_{i}, u_{\hat{\lambda}}\right)\right)+V\left(x\left(\tau ; \hat{x}_{i}, u_{\hat{\jmath}}\right)\right)-V\left(\hat{x}_{i}\right)+V\left(\hat{x}_{i}\right)-V\left(x_{i}\right) .
$$


We can bound the last two terms since $V$ is uniformly continuous in compact subsets of $\mathscr{R} \supset \Omega_{c}$. Also note that the third and forth term start from the same $\hat{x}_{i}$, and that the first term can be bound via $\alpha_{V}$ :

$$
V\left(x\left(\tau ; x_{i}, u_{\lambda}\right)\right)-V\left(x_{i}\right) \leq \alpha_{V}\left(e^{L_{f x}\left(\tau-t_{i}\right)}\left\|\hat{x}_{i}-x_{i}\right\|\right)-\int_{t_{i}}^{\tau} F\left(x\left(s ; \hat{x}_{i}, u_{\hat{\lambda}}\right), u_{\lambda}\right) d s+\alpha_{V}\left(\left\|\hat{x}_{i}-x_{i}\right\|\right)
$$

Here we used an upper bound for $\left\|x\left(\tau ; x_{i}, u_{\curlywedge}\right)-x\left(\tau ; \hat{x}_{i}, u_{\curlywedge}\right)\right\|$ based on the Gronwall-Bellman lemma. If we now assume that $x_{i} \notin \Omega_{\alpha / 2}$ and that

$$
\alpha_{V}\left(e_{\max }\right) \leq \alpha / 4
$$

then we know that $\hat{x}_{i} \notin \Omega_{\alpha / 4}{ }^{3}$. Thus we we obtain from (31) using Fact 1 that

$$
V\left(x\left(\delta ; x_{i}, u_{\curlywedge}^{\wedge}\right)\right)-V\left(x_{i}\right) \leq-V_{\min }(c, \alpha / 4, \delta)+\alpha_{V}\left(e^{L_{f x}} \delta\left\|\hat{x}_{i}-x_{i}\right\|\right)+\alpha_{V}\left(\left\|\hat{x}_{i}-x_{i}\right\|\right) .
$$

To guarantee that $x$ is decreasing from sampling instant to sampling instant along the level sets, and to achieve convergence to the set $\Omega_{\alpha / 2}$ in finite time we need that the right hand side is strictly less than zero. One possibility to obtain this is to require, that the observer parameters are chosen such that:

$$
\alpha_{V}\left(e^{L_{f x} \delta}\left\|\hat{x}_{i}-x_{i}\right\|\right)+\alpha_{V}\left(\left\|\hat{x}_{i}-x_{i}\right\|\right)-V_{\min }(c, \alpha / 4, \delta) \leq-V_{\min }(c, \alpha / 4, \delta)+\frac{1}{2} V_{\min }(c, \alpha / 4, \delta) .
$$

Thus, if we choose the observer parameters such that

$$
\alpha_{V}\left(e^{L_{f x} \delta} e_{\max }\right)+\alpha_{V}\left(e_{\max }\right) \leq \frac{1}{2} V_{\min }(c, \alpha / 4, \delta) \quad \text { and } \quad \alpha_{V}\left(e_{\max }\right) \leq \alpha / 4
$$

we achieve finite time convergence from any point $x\left(t_{i}\right) \in \Omega_{c_{0}}$ to the set $\Omega_{\alpha / 2}$.

Third part ( $\left.x\left(t_{i+1}\right) \in \Omega_{\alpha} \forall x\left(t_{i}\right) \in \Omega_{\alpha / 2}\right)$ : If $x\left(t_{i}\right) \in \Omega_{\alpha / 2}$ equation (37) is still valid. Skipping the integral contribution on the right we obtain:

$$
V\left(x\left(\tau ; x_{i}, u_{\hat{\jmath}}\right)\right)-V\left(x_{i}\right) \leq \alpha_{V}\left(e^{L_{f x}\left(\tau-t_{i}\right)}\left\|\hat{x}_{i}-x_{i}\right\|\right)+\alpha_{V}\left(\left\|\hat{x}_{i}-x_{i}\right\|\right)
$$

Thus if we assume that

$$
\alpha_{V}\left(e^{L_{f x} \delta} e_{\max }\right)+\alpha_{V}\left(e_{\max }\right) \leq \alpha / 2 .
$$

then $x\left(t_{i+1}\right) \in \Omega_{\alpha} \forall x\left(t_{i}\right) \in \Omega_{\alpha / 2}$. Combining all three steps, we obtain the theorem if

$$
\delta_{\max } \leq \min \left\{T_{c_{0} c_{1}} / k_{\text {conv }}, T_{c_{2} c}\right\} .
$$

and if we choose the observer error $e_{\max }$ such that

$$
\alpha_{V}\left(e^{L_{f x}} \delta e_{\max }\right)+\alpha_{V}\left(e_{\max }\right) \leq \min \left\{\frac{1}{2} V_{\min }(c, \alpha / 4, \delta), \alpha / 4\right\} .
$$

Remark 3.3 Explicitly designing an observer based on (40) and (39) is in general not possible. However, the theorem underpins that if the observer error can be sufficiently fast decreased that the closed-loop system state will be semiglobally practically stable.

Theorem 3.1 lays the basis for the design of observer based output feedback NMPC controllers that achieve semiglobal practical stability. While in principle Assumption 2 is difficult to satisfy, quite a number of observers designs achieve the desired properties as shown in the next Section.

\footnotetext{
${ }^{3}$ The values $\alpha / 2$ and $\alpha / 4$ are chosen for simplicity
} 


\subsection{Output Feedback NMPC with Stability-Possible Observer Designs}

Various observers satisfy Assumption 2 and thus allow the design of semi-globally stable output feedback controllers following Theorem 3.1. We will go into some detail for standard high-gain observers [87] and optimization based moving horizon observers with contraction constraint [72]. Note, that further observer designs that satisfy the assumptions are for example observers that possess a linear error dynamics where the poles can be chosen arbitrarily (e.g. based on normal form considerations and output injection $[9,51]$ ), or observers that achieve finite convergence time such as sliding mode observers [28] or the approach presented in [29].

High Gain Observers: One possible observer design approach that satisfies Assumption 2 are high-gain observers. Basically, high-gain observers obtain a state estimate based on approximated derivatives of the output signals. They are in general based on the assumption that the system is uniformly completely observable. Uniform complete observability is defined in terms of the observability map $\mathscr{H}$, which is given by successive differentiation of the output $y$ :

$$
Y^{T}=\left[y_{1}, \dot{y}_{1}, \ldots, y_{1}^{\left(r_{1}\right)}, y_{2}, \ldots, y_{p}, \ldots, y_{p}^{\left(r_{p}\right)}\right]=: \mathscr{H}(x)^{T} .
$$

Here $Y$ is the vector of output derivatives. Note that we assume for simplicity of presentation that $\mathscr{H}$ does not depend on the input and its derivatives. More general results allowing that $\mathscr{H}$ depends on the input and its derivatives can be found in [34]. We assume that the system is uniformly completely observable, i.e.

Assumption 3 The system (1) is uniformly completely observable in the sense that there exists a set of indices $\left\{r_{1}, \ldots, r_{p}\right\}$ such that the mapping $Y=\mathscr{H}(x)$ depends only on $x$, is smooth with respect to $x$ and its inverse from $Y$ to $x$ is smooth and onto.

Application of the coordinate transformation $\zeta:=\mathscr{H}(x)$, where $\mathscr{H}$ is the observability mapping, to the system (1) leads to the system in observability normal form in $\zeta$ coordinates

$$
\dot{\zeta}=A \zeta+B \phi(\zeta, u), \quad y=C \zeta .
$$

The matrices $A, B$ and $C$ have the following structure

$$
\begin{aligned}
& A=\operatorname{blockdiag}\left[A_{1}, \ldots A_{p}\right], A_{i}=\left[\begin{array}{ccccc}
0 & 1 & 0 & \cdots & 0 \\
0 & 0 & 1 & \cdots & 0 \\
\vdots & & & & \vdots \\
0 & \cdots & \ldots & 0 & 1 \\
0 & \cdots & \cdots & \cdots & 0
\end{array}\right]_{r_{i} \times r_{i}} \\
& B=\operatorname{blockdiag}\left[B_{1}, \ldots, B_{p}\right], B_{i}=\left[\begin{array}{llll}
0 & \cdots & 0 & 1
\end{array}\right]_{r_{i} \times 1}^{T} \\
& C=\operatorname{blockdiag}\left[C_{1}, \ldots, C_{p}\right], C_{i}=\left[\begin{array}{llll}
1 & 0 & \cdots & 0
\end{array}\right]_{1 \times r_{i}},
\end{aligned}
$$

and $\phi: \mathbb{R}^{n} \times \mathbb{R}^{m} \rightarrow \mathbb{R}^{p}$ is the "system nonlinearity" in observability normal form. The high-gain observer 4

$$
\dot{\hat{\zeta}}=A \hat{\zeta}+H_{\varepsilon}(y-C \hat{\zeta})+B \hat{\phi}(\hat{\zeta}, u)
$$

allows recovery of the states $[4,87] \zeta$ from information of $y(t)$ assuming that

Assumption $4 \hat{\phi}$ in (44) is globally bounded.

The function $\hat{\phi}$ is the approximation of $\phi$ that is used in the observer and which may be deduced from $\phi$. The observer gain matrix $H_{\varepsilon}$ is given by $H_{\varepsilon}=$ blockdiag $\left[H_{\varepsilon, 1}, \ldots, H_{\varepsilon, p}\right]$, with $H_{\varepsilon, i}^{T}=\left[\alpha_{1}^{(i)} / \varepsilon, \alpha_{2}^{(i)} / \varepsilon^{2}, \ldots, \alpha_{n}^{(i)} / \varepsilon^{r_{i}}\right]$, where $\varepsilon$ is the so-called high-gain parameter since $1 / \varepsilon$ goes to infinity for $\varepsilon \rightarrow 0$. The $\alpha_{j}^{(i)}$ s are design parameters and must be chosen such that the polynomials $s^{n}+\alpha_{1}^{(i)} s^{n-1}+\cdots+\alpha_{n-1}^{(i)} s+\alpha_{n}^{(i)}=0, i=1, \ldots, p$ are Hurwitz.

\footnotetext{
${ }^{4}$ We use hatted variables for the observer states and variables.
} 
Note that estimates obtained in $\zeta$ coordinates can be transformed back to the $x$ coordinates by $\hat{x}=\mathscr{H}^{-1}(\zeta)$.

As shown in [4] and utilized in [34], under the assumption that the initial observer error is out of a compact set and that the system state stays in a bounded region, for any desired $e_{\max }$ and any convergence time $k_{\mathrm{conv}} \delta$ there exists a maximum $\varepsilon^{\star}$ such that for any $\varepsilon \leq \varepsilon^{\star}$ the observer error stays bounded and satisfies: $\|x(\tau)-\hat{x}(\tau)\| \forall \tau \geq k_{\text {conv }} \delta$.

Thus, the high-gain observer satisfies Assumption 2. Further details can be found in [33,34].

Moving Horizon Observers: Moving horizon estimators (MHE) are optimization based observers, i.e. the state estimate is obtained by the solution of a dynamic optimization problem in which the deviation between the measured output and the simulated output starting from estimated initial state is minimized. Various approaches to moving horizon state estimation exist $[2,72,79,92]$. We focus here on the MHE scheme with contraction constraint as introduced in [72], since it satisfies the assumptions needed. In the approach proposed in [72] basically at all sampling instants a dynamic optimization problem is solved, considering the output measurements spanning over a certain estimation window in the past. Assuming that certain reconstructability assumptions hold and that no disturbances are present, one could in principle estimate the system state by solving one single dynamic optimization problem. However, since this would involve the solution of a global optimization problem in real-time, it is proposed in [72] to only improve the estimate at every sampling time by requiring that the integrated error between the measured output and the simulated output is decreased from sampling instant to sampling instant. Since the contraction rate directly corresponds to the convergence of the state estimation error and since it can in principle be chosen freely this MHE scheme satisfies the assumptions on the state estimator. Thus, it can be employed together with a state feedback NMPC controller to achieve semi-global practical stability as explained in Section 3.3.

\section{Conclusions}

Model predictive control for linear constrained systems has been proven as a useful control solution for many practical applications. It is expected that the use of nonlinear models in the predictive control framework, leading to nonlinear model predictive control, results in improved control performance and allows the direct use of first principle based models. However, the consideration of nonlinear models also poses challenging theoretical, computational and implementational problems.

In the first part of this paper an overview of various aspects of NMPC has been given. We mainly focused on sampled-data NMPC, since the plant model is usually derived based on first principles. As outlined, some of the challenges occurring in NMPC such as stability, efficient solution of the dynamic optimization problem etc. are already (at least partially) solved. Nevertheless many unsolved questions remain.

In the second part of the paper we addressed the important question of output feedback NMPC. Specifically we expanded the sampled-data output feedback NMPC approach for continuous time systems as presented in [33,34] to a wider class of observers stating conditions the observer error must satisfy such that the closed-loop is semi-globally practically stable. As shown, several observer designs satisfy the required conditions. However, the results should be seen as conceptual rather then as practically applicable. For example it is in general difficult to establish a priori that the value function is continuous. In general there is no guarantee that nominally stable NMPC schemes satisfy this assumption, especially if constraints on the states are present, see [40]. Thus, future research has to focus on either relaxing this condition, or to derive conditions under which an NMPC scheme does satisfy this assumption, see for example [41].

\section{References}

[1] V. Adetola and M. Guay. Nonlinear output feedback receding horizon control. In Proc. Amer. Contr. Conf., Denver, 2003.

[2] M. Alamir. Optimization based nonlinear observers revisited. Int. J. Contr., 72(13):1204-1217, 1999. 
[3] F. Allgöwer, T.A. Badgwell, J.S. Qin, J.B. Rawlings, and S.J. Wright. Nonlinear predictive control and moving horizon estimation - An introductory overview. In P.M. Frank, editor, Advances in Control, Highlights of ECC'99, pages 391-449. Springer, 1999.

[4] A.N. Atassi and H.K. Khalil. A separation principle for the stabilization of a class of nonlinear systems. IEEE Trans. Automatic Control, 44(9):1672-1687, 1999.

[5] A.N. Atassi and H.K. Khalil. Separation results for the stabilization of nonlinear systems using different highgain observer designs. Syst. Control Lett., 39(3):183-191, 2000.

[6] R.A. Bartlett, A. Wächter, and L.T. Biegler. Active set vs. inerior point strategies for model predicitve control. In Proc. Amer. Contr. Conf., pages 4229-4233, Chicago, Il, 2000.

[7] R. Bellman. Dynamic Programming. Princeton University Press, Princeton, New Jersey, 1957.

[8] A. Bemporad and A. Garulli. Output-feedback predictive control of constrained linear systems via setmembership state estimation. Int. J. Control, 73(8):655-665, 2000.

[9] D. Bestle and M. Zeitz. Canonical form observer design for non-linear time-variable systems. Int. J. Contr., 38(2):419-431, 1983.

[10] L. Biegler. Efficient solution of dynamic optimization and NMPC problems. In F. Allgöwer and A. Zheng, editors, Nonlinear Predictive Control, pages 219-244. Birkhäuser, 2000.

[11] L.T. Biegler and J.B Rawlings. Optimization approaches to nonlinear model predictive control. In W.H. Ray and Y. Arkun, editors, Proc. 4th International Conference on Chemical Process Control - CPC IV, pages 543-571. AIChE, CACHE, 1991.

[12] T. Binder, L. Blank, H.G. Bock, R. Burlisch, W. Dahmen, M. Diehl, T. Kronseder, W. Marquardt, J.P. Schlöder, and O. von Stryk. Introduction to model based optimization of chemical processes on moving horizons. In M. Groetschel, S.O. Krumke, and J. Rambau, editors, Online Optimization of Large Scale Systems: State of the Art, pages 295-339. Springer, 2001.

[13] R. Blauwkamp and T. Basar. A receding-horizon approach to robust output feedback control for nonlinear systems. In Proc. 38th IEEE Conf. Decision Contr, pages 4879-4884, San Diego, 1999.

[14] M. Cannon, B. Kouvaritakis, Y. I. Lee, and A. C. Brooms. Efficient non-linear model based predictive control. Int. J. Control, 74(4):361-372, 2001.

[15] C.C. Chen and L. Shaw. On receding horizon feedback control. Automatica, 18(3):349-352, 1982.

[16] H. Chen. Stability and Robustness Considerations in Nonlinear Model Predictive Control. Fortschr.-Ber. VDI Reihe 8 Nr. 674. VDI Verlag, Düsseldorf, 1997.

[17] H. Chen and F. Allgöwer. Nonlinear model predictive control schemes with guaranteed stability. In R. Berber and C. Kravaris, editors, Nonlinear Model Based Process Control, pages 465-494. Kluwer Academic Publishers, Dodrecht, 1998.

[18] H. Chen and F. Allgöwer. A quasi-infinite horizon nonlinear model predictive control scheme with guaranteed stability. Automatica, 34(10):1205-1218, 1998.

[19] H. Chen, C.W. Scherer, and F. Allgöwer. A game theoretic approach to nonlinear robust receding horizon control of constrained systems. In Proc. Amer. Contr. Conf., pages 3073-3077, Albuquerque, 1997.

[20] W. Chen, D.J. Ballance, and J. O'Reilly. Model predictive control of nonlinear systems: Computational delay and stability. IEE Proceedings, Part D, 147(4):387-394, 2000. 
[21] L. Chisci, , and G. Zappa. Feasibility in predictive control of constrained linear systems: the output feedback case. Int. J. of Robust and Nonlinear Control, 12(5):465-487, 2002.

[22] G. De Nicolao, L. Magni, and R. Scattolini. Stability and robustness of nonlinear receding horizon control. In F. Allgöwer and A. Zheng, editors, Nonlinear Predictive Control, pages 3-23. Birkhäuser, 2000.

[23] N. M.C. de Oliveira and L. T. Biegler. An extension of Newton-type algorithms for nonlinear process control. Automatica, 31(2):281-286, 1995.

[24] S. de Oliveira Kothare and M. Morari. Contractive model predictive control for constrained nonlinear systems. IEEE Trans. Automat. Contr., 45(6):1053-1071, 2000.

[25] M. Diehl, R. Findeisen, Z. Nagy, H.G. Bock, J.P. Schlöder, and F. Allgöwer. Real-time optimization and nonlinear model predictive control of processes governed by differential-algebraic equations. J. Proc. Contr., 4(12):577$585,2002$.

[26] M. Diehl, R. Findeisen, S. Schwarzkopf, I. Uslu, F. Allgöwer, and J.P. Bock, H.G. Schlöder. An efficient approach for nonlinear model predictive control of large-scale systems. Part I: Description of the methodology. Automatisierungstechnik, 12:557-567, 2002.

[27] M. Diehl, R. Findeisen, S. Schwarzkopf, I. Uslu, F. Allgöwer, and J.P. Bock, H.G. Schlöder. An efficient approach for nonlinear model predictive control of large-scale systems. Part II: Experimental evaluation considering the control of a distillation column. Automatisierungstechnik, 1:22-29, 2003.

[28] S. Drakunov and V. Utkin. Sliding Mode Observers. Tutorial. In Proc. 34th IEEE Conf. Decision Contr., pages 3376-3378, New Orleans, LA, December 1995. IEEE.

[29] R. Engel and G. Kreisselmeier. A continuous-time observer which converges in finite time. IEEE Trans. Aut. Control, 47(7):1202-1204, 2002.

[30] R. Findeisen. Stability, Computational Efficiency, Robustness, and Output Feedback in Sampled-Data Nonlinear Model Predictive Control. PhD thesis, 2003. PhD thesis, University of Stuttgart, in preparation.

[31] R. Findeisen and F. Allgöwer. The quasi-infinite horizon approach to nonlinear model predictive control. In A. Zinober and D. Owens, editors, Nonlinear and Adaptive Control, Lecture Notes in Control and Information Sciences, pages 89-105, Berlin, 2001. Springer-Verlag.

[32] R. Findeisen, M. Diehl, I. Uslu, S. Schwarzkopf, F. Allgöwer, H.G. Bock, J.P. Schlöder, and Gilles. Computation and performance assesment of nonlinear model predictive control. In Proc. 42th IEEE Conf. Decision Contr., Las Vegas, USA, 2002.

[33] R. Findeisen, L. Imsland, F. Allgöwer, and B.A. Foss. Output feedback nonlinear predictive control - a separation principle approach. In Proceedings of 15th IFAC World Congress, Barcelona, Spain, 2002.

[34] R. Findeisen, L. Imsland, F. Allgöwer, and B.A. Foss. Output feedback stabilization for constrained systems with nonlinear model predictive control. Int. J. of Robust and Nonlinear Control, 13(3-4):211-227, 2003.

[35] R. Findeisen, Z. Nagy, M. Diehl, F. Allgöwer, H.G. Bock, and J.P. Schlöder. Computational feasibility and performance of nonlinear model predicitve control. In Proc. 6st European Control Conference ECC'01, pages 957-961, Porto, Portugal, 2001.

[36] F.A. Fontes. A general framework to design stabilizing nonlinear model predictive controllers. Syst. Contr. Lett., 42(2):127-143, 2000.

[37] F.A. Fontes. Discontinuous feedbacks, discontinuous optimal controls, and continuous-time model predictive control. Int. J. of Robust and Nonlinear Control, 13(3-4):191-209, 2003. 
[38] J. B. Froisy. Model predictive control: Past, present and future. ISA Transactions, 33:235-243, 1994.

[39] C.E. García, D.M. Prett, and M. Morari. Model Predictive Control: Theory and practice - A survey. Automatica, 25(3):335-347, 1989.

[40] G. Grimm, M.J. Messina, A.R. Teel, and S. Tuna. Examples when model predictive control is nonrobust. submitted, 2002.

[41] G. Grimm, M.J. Messina, A.R. Teel, and S. Tuna. Model predictive control: For want of a local control Lyapunov function, all is not lost. submitted, 2002.

[42] L. Imsland, R. Findeisen, F. Allgöwer, and B.A. Foss. Output feedback stabilization with nonlinear predictive control - asymptotic properties. In Proc. Amer. Contr. Conf., Denver, 2003.

[43] L. Imsland, R. Findeisen, E. Bullinger, F. Allgöwer, and B.A. Foss. A note on stability, robustness and performance of output feedback nonlinear model predictive control. To appear in J. Proc. Contr., 2002.

[44] L. Imsland, R. Findeisen, E. Bullinger, F. Allgöwer, and B.A. Foss. On output feedback nonlinear model predictive control using high gain observers for a class of systems. In 6th IFAC Symposium on Dynamics and Control of Process Systems, DYCOPS-6, pages 91-96, Jejudo, Korea, 2001.

[45] A. Jadbabaie, J. Yu, and J. Hauser. Unconstrained receding horizon control of nonlinear systems. IEEE Trans. Automat. Contr., 46(5):776-783, 2001.

[46] S.S. Keerthi and E.G. Gilbert. An existence theorem for discrete-time infinite-horizon optimal control problems. IEEE Trans. Automat. Contr., 30(9):907-909, 1985.

[47] S.S. Keerthi and E.G. Gilbert. Optimal infinite-horizon feedback laws for a general class of constrained discretetime systems: Stability and moving-horizon approximations. J. Opt. Theory and Appl., 57(2):265-293, 1988.

[48] C. Kellett, H. Shim, and A. Teel. Robustness of discontinuous feedback via sample and hold. In Proc. Amer. Contr. Conf., pages 3512-3516, Anchorage, 2002.

[49] M.V. Kothare, V. Balakrishnan, and M. Morari. Robust constrained model predictive control using linear matrix inequalities. Automatica, 32(10):1361-1379, 1996.

[50] B. Kouvaritakis, W. Wang, and Y. I. Lee. Observers in nonlinear model-based predictive control. Int. J. of Robust and Nonlinear Control, 10(10):749-761, 2000.

[51] A.J. Krener and A. Isidori. Linearization by output injection and nonlinear observers. Syst. Contr. Lett., 3:47-52, 1983.

[52] S. Lall and K. Glover. A game theoretic approach to moving horizon control. In D. Clarke, editor, Advances in Model-Based Predictive Control. Oxford University Press, 1994.

[53] J.H. Lee and B. Cooley. Recent advances in model predictive control and other related areas. In J.C. Kantor, C.E. Garcia, and B. Carnahan, editors, Fifth International Conference on Chemical Process Control - CPC V, pages 201-216. American Institute of Chemical Engineers, 1996.

[54] Y.I. Lee and B. Kouvaritakis. Receding horizon output feedback control for linear systems with input saturation. IEE Control Theory Appl., 148(2):109-115, 2001.

[55] W.C. Li and L.T. Biegler. Multistep, newton-type control strategies for constrained nonlinear processes. Chem. Eng. Res. Des., 67:562-577, 1989.

[56] J. Löfberg. Towards joint state estimation and control in minimax MPC. In Proceedings of 15th IFAC World Congress, Barcelona, Spain, 2002. 
[57] L. Magni, D. De Nicolao, and R. Scattolini. Output feedback receding-horizon control of discrete-time nonlinear systems. In Preprints of the 4th Nonlinear Control Systems Design Symposium 1998 - NOLCOS'98, pages 422-427. IFAC, July 1998.

[58] L. Magni, G. De Nicolao, R. Scatollini, and F. Allgöwer. Robust model predictive control for nonlinear discretetime systems. Int. J. of Robust and Nonlinear Control, 13(3-4):229-246, 2003.

[59] L. Magni, G. De Nicolao, and R. Scattolini. Output feedback and tracking of nonlinear systems with model predictive control. Automatica, 37(10):1601-1607, 2001.

[60] L. Magni, G. De Nicolao, and R Scattolini. A stabilizing model-based predicitve control algorithm for nonlinear systems. Automatica, 37(10):1351-1362, 2001.

[61] L. Magni, G. De Nicolao, R. Scattolini, and F. Allgöwer. Robust receding horizon control for nonlinear discretetime systems. In Proceedings 15th IFAC World Congress, Barcelona, Spain, 2001.

[62] L. Magni, H. Nijmeijer, and A.J. van der Schaft. A receding-horizon approach to the nonlinear $\mathrm{H}_{\infty}$ control problem. Automatica, 37(5):429-435, 2001.

[63] L. Magni and R. Scattolini. State-feedback MPC with piecewise constant control for continuous-time systems. In Proc. 42th IEEE Conf. Decision Contr., Las Vegas, USA, 2002.

[64] L. Magni and R. Sepulchre. Stability margins of nonlinear receding-horizon control via inverse optimality. Syst. Contr. Lett., 32(4):241-245, 1997.

[65] R. Mahadevan and F.J. Doyle III. Efficient optimization approaches to nonlinear model predictive control. Int. J. of Robust and Nonlinear Control, 13(3-4):309-329, 2003.

[66] F. Martinsen, L.T. Biegler, and B.A Foss. Application of optimization algorithms to nonlinear mpc. In Proceedings of 15th IFAC World Congress, Barcelona, Spain, 2002.

[67] D.Q. Mayne. Optimization in model based control. In Proc. IFAC Symposium Dynamics and Control of Chemical Reactors, Distillation Columns and Batch Processes, pages 229-242, Helsingor, 1995.

[68] D.Q. Mayne and H. Michalska. Receding horizon control of nonlinear systems. IEEE Trans. Automat. Contr., 35(7):814-824, 1990.

[69] D.Q. Mayne, J.B. Rawlings, C.V. Rao, and P.O.M. Scokaert. Constrained model predictive control: stability and optimality. Automatica, 26(6):789-814, 2000.

[70] E.S. Meadows, M.A. Henson, J.W. Eaton, and J.B. Rawlings. Receding horizon control and discontinuous state feedback stabilization. Int. J. Contr., 62(5):1217-1229, 1995.

[71] H. Michalska and D.Q. Mayne. Robust receding horizon control of constrained nonlinear systems. IEEE Trans. Automat. Contr., AC-38(11):1623-1633, 1993.

[72] H. Michalska and D.Q. Mayne. Moving horizon observers and observer-based control. IEEE Trans. Automat. Contr., 40(6):995-1006, 1995.

[73] M. Morari and J.H. Lee. Model predicitve control: Past, present and future. Comp. and Chem. Eng., 23(4/5):667$682,1999$.

[74] K.R. Muske, E.S. Meadows, and J.R. Rawlings. The stability of constrained receding horizon control with state estimation. In Proc. Amer. Contr. Conf., pages 2837-2841, Baltimore, 1994.

[75] J. Primbs, V Nevistić, and J. Doyle. Nonlinear optimal control: A control Lyapunov function and receding horizon perspective. Asian Journal of Control, 1(1):14-24, 1999. 
[76] R. Pytlak. Numerical Methods for Optimal Control Problems with State Constraints. Lecture Notes in Mathematics. Springer, Berlin, 1999.

[77] S.J. Qin and T.A. Badgwell. An overview of nonlinear model predictive control applications. In F. Allgöwer and A. Zheng, editors, Nonlinear Predictive Control, pages 369-393. Birkhäuser, 2000.

[78] S.J. Qin and T.A. Badgwell. A survey of industrial model predictive control technology. Accepted for Control Engineering Practice, 2003.

[79] C. V. Rao, J. B. Rawlings, and D. Q. Mayne. Constrained state estimation for nonlinear discretetime systems: Stability and moving horizon approximations. IEEE Trans. Automat. Contr., 48(2):246-258, 2003.

[80] J. B. Rawlings. Tutorial overview of model predictive control. IEEE Contr. Syst. Magazine, 20(3):38-52, 2000.

[81] J.B. Rawlings, E.S. Meadows, and K.R. Muske. Nonlinear model predictive control: A tutorial and survey. In Proc. Int. Symp. Adv. Control of Chemical Processes, ADCHEM, Kyoto, Japan, 1994.

[82] P.O.M. Scokaert, D.Q. Mayne, and J.B. Rawlings. Suboptimal model predictive control (feasibility implies stability). IEEE Trans. Automat. Contr., 44(3):648-654, 1999.

[83] P.O.M. Scokaert, J.B. Rawlings, and E.S. Meadows. Discrete-time stability with perturbations: Application to model predictive control. Automatica, 33(3):463-470, 1997.

[84] M. Sznaier, R. Suarez, and J. Cloutier. Suboptimal control of constrained nonlinear systems via receding horizon control Lypunov functions. Int. J. of Robust and Nonlinear Control, 13(3-4):247-259, 2003.

[85] A. Teel and L. Praly. Tools for semiglobal stabilization by partial state and output feedback. SIAM J. Control and Optimization, 33(5):1443-1488, 1995.

[86] M.J. Tenny and J.B. Rawlings. Feasible real-time nonlinear model predictive control. In 6th International Conference on Chemical Process Control - CPC VI, AIChE Symposium Series, 2001.

[87] A. Tornambè. Output feedback stabilization of a class of non-miminum phase nonlinear sysems. Syst. Contr. Lett., 19(3):193-204, 1992.

[88] Z. Wan and M.V. Kothare. Robust output feedback model predictive control using offline linear matrix inequalities. J. Proc. Contr., 12(7):763-774, October 2002.

[89] Z. Wan and M.V. Kothare. Efficient stabilizing output feedback model predictive control for constrained nonlinear systems. In Proc. Amer. Contr. Conf., 2003.

[90] S. J. Wright. Applying new optimization algorithms to model predictive control. In J.C. Kantor, C.E. Garcia, and B. Carnahan, editors, Fifth International Conference on Chemical Process Control - CPC V, pages 147-155. American Institute of Chemical Engineers, 1996.

[91] A. Zheng and M. Morari. Stability of model predictive control with mixed constraints. IEEE Trans. Automat. Contr., AC-40(10):1818-1823, 1995.

[92] G. Zimmer. State observation by on-line minimization. Int. J. Contr., 60(4):595-606, 1994. 